Theories \& Applications, the International Edition

Printed Version: (ISSN 2090-5262)

Online Version: (ISSN 2090-5270)

November 2012, Volume 2, No. 3 Pages (17 - 30)

\title{
The Basic Physical Characteristics of High Level Rowers
}

\author{
Mohamed Ashraf Abdel-Sattar Awad*
}

\begin{abstract}
:
The physical measurements are highly related to the athletic field. Every athletic activity has special physical requirements distinguish it from other activities. These requirements reflected on the physical characteristics that anyone who practices any specific activity should have so that he could reach the highest levels in the practiced activity. The availability of the appropriate physical measurements data is important as an indicator that must be available so that the players could reach the highest possible athletic level. The research aims at identifying the basic physical characteristics of high level rowers. The research sample was randomly selected from rowers enrolled in the records of the Egyptian Rowing Federation for the season 2011. The sample consisted of 18 rowers from the rowing clubs in Alexandria and Ismailia, and was selected based registraion in the records of the Egyptian Rowing Federation, participation in competitions for no less than two consecutive years and winning one of the top three positions in the adult rower competitions. The study recommends using the selected battery of physical measurements for rowers to develop the activity through improving the selection by using the battery and the standard grades extracted, and upgrading training operations by improving the level of maximum strength, especially for the legs. When selecting rowers, the following measurements should be considered: height - sitting height - forearm and palm length - ratio of the legs length - the grip strength the maximum strength of the legs. Training should increase the relative strength of the muscles of the body through muscle strength training, especially for the legs, raising the level of the relative strength of the body as a whole.
\end{abstract}

\section{Introduction and Research Problem:}

Body measurements are closely related to sport. Each sport activity has its own physical requirements distinguishing it from other activities. These requirements are reflected on the physical and somatic characteristics that should be possessed by anyone before attempting to reach higher levels in one particular sport. Data on body measurements has gained importance as a necessary indicator of the possibility of reaching higher possible levels in sport.

In this regard, Kamal Abd el-Hamid Ismail (2003) mentioned that anthropometry is an important branch of knowledge and is closely related to the study of measurement in physical education. It has actually contributed to the

\footnotetext{
* Assistant Professor, Faculty of Physical Education, Tanta University, Egypt.
}

field of sport and helps the coach select the physical measurements appropriate for the practice of a specific sport activity, which, in turn enables a player to reach higher levels. (5: 236)

According to Essam Abdul-Khaliq Mustafa (1994), anthropometry is a relatively new scientific field, yet it has already occupied a prominent position in scientific sport research. It is like a mirror that reflects the relationship between the body shape and the skillful performance of a person. (3:8)

Commenting on the importance of physical measurements in the field of sport, Osama Kamel Rateb and Ali Mohammed Zaki (1992) stated that physical measurements affect the efficiency of sport performance and have an impact on the physical, skillful and mechanical aspects too. The human bodies differ in terms of shape, size as well as physical capacities. Recent sports research tends to determine the 
special physical characteristics required for each sport, so that the selection of junior players is based on science. Such a selection would contribute to excellence in sport while saving time and effort. (1: 309)

According to Mikuliae (2008), there is quite a few studies on anthropometric characteristics, such as height, weight and limb lengths of rowers. (16:12)

Niels and Steamos (2007) define rowing as "one of the water sports which are practiced in an aqueous medium using boats and boat equipments, such as free - design paddles. Through the players' movements, the boat covers a distance in the least possible time according to competition rules in order to achieve the objectives of the sport activity. $(17: 11)$

Quoting Williams, Siberian, and Ropson and Mohamed Sabry Omar (1981) states that physical measurements, such as weight, height, length of limbs and muscular strength in rowing are important. He also stresses the fact that physical measurements and muscular strength are the most important potentials related to performance in rowing and designing the tools. (8: 90)

Fell, J. W. and P.T. Gaffney (2001) indicated that studies made on rowers showed that they are taller and heavier than the athletes in other sports. The anthropometric characteristics of rowers are important in directing the talented males and females alike. (11: 188). This was also indicated by Shephard, R. J. (1998) and Secher \& Vaage (1983). (20: 603) (19: 88)

Rodriguez (1986) adds that the values of length of limbs in rowers are absolute and not relative. (18: 255)

Bearing in mind the importance of body measurements in sport in general, and in rowing in particular, and the nature of the rowing sport which needs special body measurements, as a determinant of science-based selection, the researcher conducted this research in order to determine the special physical measurements for high level rowers in the Arab Republic of Egypt. As far as the author of this current study is aware, no study has so far been made to determine the physical characteristics of the Egyptian high level rowers so that the basic components and the general characteristics of the physical measurements of the said players can be identified, so as to facilitate the comparison and selection processes.

\section{Research Objective:}

The research aims at identifying the basic physical characteristics of high level rowers.

\section{Research Questions:}

The research attempted to answer the following question: What are the basic physical characteristics of high level rowers? In order to identify the absolute characteristics of elite Egyptian rowers, factorial analysis was conducted on their measurements, thus describing the factorial structure of the somatic characteristics and muscular strength which have not been addressed so far. Such characteristics, and results elicited from them, exclusively apply to Egyptians and are not comparable to those of other peoples with different anthropometric characteristics.

\section{Importance of the Research:}

The rower's body plays the central role in the rowing sport and is used to achieve the aim of the movement. It performs the movement directly, using the body parts, or indirectly, utilizing rowing aids, to achieve the aim of the rower. The physical potentials and capabilities of the player influence his ability to achieve the goal of the movement.

Physical measurements are important as requirements for the kinetic performance in rowing. In order to reach higher levels, rowers must have specific physical measurements and potentials.

Hunsicker (1974) gave the somatotype and anthropometry a paramount importance after strength and endurance as factors affecting sport performance efficiency because of their different effects associated with physical, mechanical and skillful aspects. (14: 349)

The current research attempts to identify a number of the basic physical measurements of rowers with high sport levels, that may contribute to the development and planning of 
training programs appropriate to the capacities of the rowers, especially the physical capacities. Physical measurements are relatively reliable, and are described as being predictive attributes because of their relative reliability in theories of selection. Such reliability ensures measurements necessary for selection in the rowing sport and helps select the best rowers based on scientific foundations. This in turn would contribute to excellence sport while saving time and effort.

\section{Previous Studies:}

In their study entitled "The Relative Importance of the Physical Measurements and their Relationship to the Speed of Front Crawl Swimming in Short Distances Female Swimmers", Mohamed Sabry Omar and Sanaa Hassan Al-Jubeili (1988) aimed at determining the relative importance of the physical measurements, and finding the predictive equation of the most important physical measurements affecting the speed of swimming in short distances female swimmers. Taking the physical measurements of a sample of 50 short distances female swimmers from different ages, the authors came to the conclusion that the major characteristics of the study sample were: swimmers had longer upper arm, were lighter in weight, had greater foot surface and foot area and had shorter legs. The study recommended taking these physical measurements into account when selecting short distances female swimmers. (9)

The study by Hamid Arazi et al (2011), "Anthropometric and Physiological Profiles of Elite Iranian Junior Rowers", identified the anthropometric and functional characteristics of Iranian male and female high level junior rowers. Measurements were taken of a sample of 33 male and 33 female players in the national camp of training. Research resulted in computing the arithmetic means for the following measurements; height, weight, upper limb length, lower limb length, shoulders breadth, elbow breadth, knee breadth, tarsus breadth, skinfolds, standing height and sitting height. Means for males and females respectively were; $(181.1 \pm 4.9-170.3 \pm 4.1$ $\mathrm{cm}),(80.6 \pm 3.9-70.1 \pm 4.4 \mathrm{~kg}),(49.4 \pm 3.7-$ $44.3 \pm 4.4 \mathrm{~cm}),(104.5 \pm 4.7-93.2 \pm 5.7 \mathrm{~cm})$, $(55.85 \pm 3.9-40.1 \pm 3.5 \mathrm{~cm}),(30.1 \pm 3.2-24.5$ $\pm 2.3 \mathrm{~cm}),(39.16 \pm 2.7-36.5 \pm 2.5 \mathrm{~cm}),(27.1 \pm$ $2.5-23.5 \pm 1.8 \mathrm{~cm}),(10.6 \pm 3.6-15.7 \pm 3.8$ $\mathrm{cm}),(29.1 \pm 14.1-19.3 \pm 9.5$ repetition $),(66.0$ $\pm \quad 8.5 \quad-59.0 \pm 9.7$ repetition) The research recommended using these measurements and characteristics in selecting and training in rowing. 13.

The study by S. Kaloupsis et al (2008) entitled "Anthropometric Characteristics and Somatotype of Young Greek Rowers" aimed at identifying the anthropometric characteristics of the young rowers by comparing them with international rowers and non-practicing children. Body and somotatotype measurements were taken of a sample of 29 players in the age group of 11-16 years old. One of the most important results was the presence of significant differences, especially in the age group of 11-16 years old. The research recommended using the measurements extracted in selecting rowers in the same age group. There were significant differences, favoring the 21 year-old group, in somatotypes, heights and weights (21).

The study of G. J. Slater et al (2012) entitled "Physique traits of light weight rowers and their relationship to competitive success" aimed at identifying the morphological characteristics of rowing light weight rowers who won the rowing Australian championship. All physical measurements have been taken of the research sample of 107 male light weight rowers under the age of 23 years and 45 female rowers. Those who did not complete their data or left the open competition were excluded. The performance of 66 rowers in the single boat competitions was also evaluated and body measurements were taken during the tournament. The main result of the study was that the success factors of the performance were associated with a lower level of body fat and higher values of body mass, muscle mass and height. The study recommended taking these elements into account during selection, and reducing the body fat level and increasing the muscle mass during training. (12)

The study of Kerr et al, (2007) of "Common anthropometric characteristics of Olympic rowers", aimed at identifying the common anthropometric characteristics of Olympic rowers in the light weight and open weight categories. An anthropometric test battery was 
used to measure 38 anthropometric dimensions of a sample of 140 male rowers in open weight category, 69 female rowers of the same category, 50 male rowers in the lightweight category and 14 female rowers in the same category. The main results showed the presence of significant differences between males and females in the two categories of light weight and open weight categories of rowers in height, wrist breadth, height of the pelvis, sitting height and grip strength. (15)

\section{Research Procedures:}

\section{Methodology:}

The descriptive method was used, being suitable for this type of study.

\section{Sample:}

The research sample was randomly selected from rowers enrolled in the records of the Egyptian Rowing Federation for the 2011 2012 season. The sample consisted of 18 rowers from the rowing clubs in Alexandria and Ismailia.

Sample was selected based on the following conditions:

- Registration in the records of the Egyptian Rowing Federation.

- Participation in competitions for no less than two consecutive years.
- Winning one of the top three positions in the adult rower competitions.

- The use of factorial analysis. It is to be noted that several studies, worldwide, have used factorial analysis on samples with population smaller than that used in this current study. In this respect, the Linkhood Method was used.

\section{Data Collecting Tools:}

- Calibrated medical balance to measure weight.

- Restameter to measure height.

- Grip dynamometer to measure grip strength.

- Dynamometer to measure the strength of feet and back muscles.

- Cable testometer to measure arm strength.

- A measuring tape.

- Data registration form.

\section{Statistical Treatment:}

Statistical treatments were made using the computer software SPSS V 20. The mean, median, mode, standard deviation and kurtosis $\&$ skewness coefficients of the study variables were calculated.

Factorial analysis was also done in the basic components manner using the Kaiser test. Factors saturated for more than 3 variables not less than \pm 0.3 were accepted. 
Results:

Table (1)

Central tendency and standard deviation measurements and measurements of the form of distribution of the research variables

\begin{tabular}{|c|c|c|c|c|c|c|}
\hline \multirow{2}{*}{ Variables } & \multicolumn{3}{|c|}{ Central tendency measurements } & \multirow{2}{*}{$\begin{array}{l}\text { Standard } \\
\text { deviation }\end{array}$} & \multicolumn{2}{|c|}{ Form of distribution } \\
\hline & Mean & Median & Mode & & Kurtosis & Skewness \\
\hline weight & 72.4520 & 72.0450 & 70.04 & 4.56806 & 0.118 & 0.416 \\
\hline total height & 173.0000 & 171.2500 & 168.00 & 5.71548 & 1.089 & 0.703 \\
\hline sitting height & 87.1500 & 88.0000 & 86.00 & 2.40428 & 0.275 & 0.977 \\
\hline arm length & 83.2000 & 81.5000 & 81.00 & 4.30245 & 1.110 & 0.476 \\
\hline upper arm length & 33.8500 & 34.0000 & 33.00 & 2.08233 & 0.413 & 0.379 \\
\hline forearm and palm length & 48.9000 & 49.7500 & 50.00 & 3.16930 & 1.031 & 0.053 \\
\hline lower limb length & 86.15000 & 85.2500 & 80.00 & 5.89279 & 1.192 & 0.559 \\
\hline foot length & 89.3000 & 88.5000 & 88.50 & 3.83116 & 0.421 & 0.273 \\
\hline thigh length & 35.9000 & 37.0000 & 37.00 & 2.53640 & 1.448 & 0.421 \\
\hline leg length & 45.9500 & 45.2500 & 45.00 & 2.26630 & 0.149 & 0.811 \\
\hline ratio of sitting height & 51.1260 & 51.3200 & 47.25 & 3.52723 & 1.924 & 1.380 \\
\hline ratio of arm length & 47.7000 & 47.7850 & 44.51 & 1.81326 & 0.361 & 0.389 \\
\hline ratio of foot length & 51.6230 & 51.8450 & 52.68 & 1.50813 & 0.489 & 1.257 \\
\hline right grip strength & 39.4000 & 38.0000 & 38.00 & 5.58172 & 0.225 & 0.479 \\
\hline left grip strength & 37.2000 & 36.0000 & 30.00 & 6.87669 & 0.372 & 0.997 \\
\hline back strength & 93.5000 & 97.5000 & 80.00 & 14.91643 & 1.343 & 0.207 \\
\hline feet strength & 104.0000 & 105.0000 & 105.00 & 22.70585 & 0.102 & 0.144 \\
\hline arms and palm strength & 96.5000 & 97.5000 & 80.00 & 12.03005 & 1.083 & 0.072 \\
\hline relative right grip & 00.5440 & 0.5550 & 0.46 & 00.06381 & 0.143 & 0.383 \\
\hline relative left grip & 00.5160 & 0.4850 & 0.43 & 00.09228 & 0.409 & 0.868 \\
\hline relative back strength & 1.2880 & 1.2950 & 1.14 & 00.18042 & 1.548 & 0.007 \\
\hline relative feet strength & 1.4290 & 1.4900 & 1.50 & 00.26539 & 0.116 & 0.125 \\
\hline relative arms strength & 1.3340 & 1.3200 & 1.10 & 00.15714 & 0.727 & 0.321 \\
\hline
\end{tabular}

Table (1) shows that the kurtosis and skewness coefficients reflect the moderation of the frequent distribution of the variables, thus allowing factor analysis. 


\begin{tabular}{|c|c|c|c|c|c|c|c|c|c|c|c|c|c|c|c|c|c|c|c|c|c|c|c|}
\hline 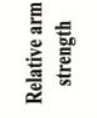 & gి & $\stackrel{\circ}{\circ}$ & \% & 萦 & व్రి & * $\bar{g}$ & g్ & $\frac{\bar{m}}{0}$ & $\approx \frac{\sigma}{0}$ & * & 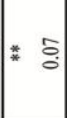 & * & $*$ s. & 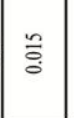 & 高 & 高 & $\frac{\overline{9}}{0}$ & $\mid \bar{s}$ & * & 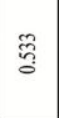 & $\begin{array}{l}\infty \\
6 \\
b \\
0\end{array}$ & ఫ্ণ্ণ & $*-$ \\
\hline 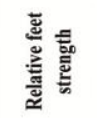 & \#年 & 。্. & 蒙 & 랑 & $\approx \approx$ & ঞ̊ㅁㅇ & * : & $* \frac{\infty}{0}$ & 응 & * : & $=\frac{n}{0}$ & สี่ & $\overline{\text { ă }}$ & हु & 声 & ఫ̊ర్త & है & . & $\stackrel{\infty}{3}$ & 。్ల్ & $\begin{array}{l}\infty \\
: 0 \\
0\end{array}$ & - & ఫ్రి \\
\hline 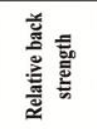 & E. & \# & శ్రీ & 壱 & 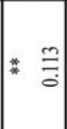 & $\bar{\Xi}$ & $*$ 홍 & * $\frac{8}{\circ}$ & 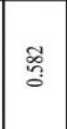 & สี & $\approx$ 象 & $\overline{0}$ & 气̂̀. & * & 5 & * & $\approx$ : & $\begin{array}{lll}* & 0 \\
& 0 \\
:\end{array}$ & 离 & छे & - & * ${ }^{*} \begin{array}{c}\infty \\
\vdots \\
0\end{array}$ & * \\
\hline 离 & $*:$ & $\approx$ * & 怘 & * & \begin{tabular}{|l|} 
\\
品
\end{tabular} & $\stackrel{\infty}{0}$ & * & 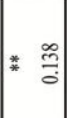 & हี & * $\overline{\text { ( }}$ & $\frac{m}{0}$ & శี & 竎 & . & * & ํㅡㅇ & å & * & $\begin{array}{l}\text { L } \\
\text { E. }\end{array}$ & - & పे & 离 & $*$ * \\
\hline 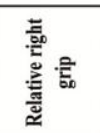 & $\frac{\hat{2}}{0}$ & $\frac{\infty}{0}$ & F & สี & * & $\#$ : & 筞 & * 궁 & $\stackrel{\infty}{\circ}$ & * & స్త్ & $\neq \frac{\infty}{6}$ & तू & ఃे & 免 & हू & 商 & \# & - & 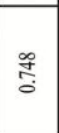 & $\begin{array}{l}0 \\
\vdots \\
\vdots \\
0\end{array}$ & $*^{*} \stackrel{\infty}{0}$ & 营 \\
\hline 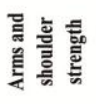 & ते & 声 & 哭 & 导 & 翏 & . * 篣 & 章 & $\overline{\mathrm{O}}$ & $\approx$ 壳 & * & $\approx$ : & * $\overrightarrow{\mathrm{O}}$ & $: \pm$ & శ్ & $\ddot{\circ}$ & 荧 & శ్ क్ & - & * & 草 & 苂 & \begin{tabular}{|l|} 
\\
\\
\end{tabular} & $*$ * \\
\hline 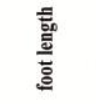 & 等 & స్ & $\stackrel{0}{0}$ & 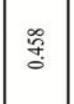 & $\overline{\mathrm{S}}$ & $* \frac{5}{0}$ & $\underset{\bar{\alpha}}{\bar{\alpha}}$ & - & $\#$ 寒 & * & $=$ : & * & * क హి & : & : & క్ & 응 & 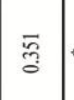 & ${ }^{*}$ ) & $\stackrel{\infty}{0}$ & $\stackrel{\circ}{\circ}$ & $\frac{\infty}{0}$ & $\frac{0}{0}$ \\
\hline 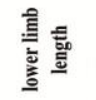 & $\approx \vec{a}$ & $\overline{\mathrm{g}}$ & ส్రీ & 鹿 & 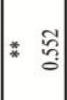 & 表 & $*-$ & $\#$ \# & 志 & * & 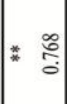 & t⿱艹⿸⿻一丿口子⿴囗十 & $\stackrel{\circ}{0}$ & हे & 菅 & $\frac{\mathrm{t}}{0}$ & $\frac{5}{0}$ & 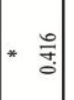 & 高 & $\overline{\bar{c}}$ & $\vec{E}$ & छे & g̊ \\
\hline 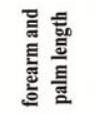 & छे & $\# \frac{9}{8}$ & 夈 & 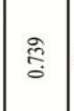 & $\#$ : & - & * & $=\frac{5}{8}$ & 啇 & ఫृ: & $\# \stackrel{\vdots}{0}$ & $\underset{0}{~}$ & : & $*$ * & $\frac{\tilde{m}}{0}$ & $=\frac{0}{0}$ & * & * & 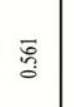 & $\stackrel{\circ}{0}$ & $\vec{E}$ & $\approx \approx$ & 㐟 \\
\hline 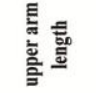 & $\approx \approx$ & $=$ : స్ & స్త్రీ & * & - & ले & * $*$ ถู & * : & ฌ̊ ప్ & 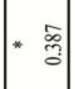 & 硧 & 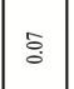 & 今. & * & * & $\frac{t}{3}$ & $\stackrel{0}{\circ}$ & $*$ * & 范 & $\stackrel{\circ}{\sharp}$ & $\stackrel{\cong}{0}$ & o. & ఫ્ \\
\hline 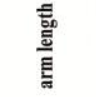 & gू & $\frac{n}{3}$ & $\cong$ & - & * & $\approx$ : & 瓷 & $\left.\begin{array}{|l|l}* \\
*\end{array}\right)$ & $\overline{\check{d}}$ & $\approx$ : & $\frac{9}{0}$ & $\approx$ * & $\frac{\mathrm{m}}{\mathrm{z}}$ & $\frac{8}{0}$ & క్ర & ๕̊̆ & సેં & $\approx$ 年 & శ్ & 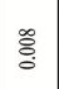 & $\frac{9}{8}$ & * 동 & * $\quad$ 索 \\
\hline 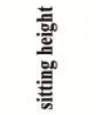 & ¿े & छ্টి & - & $\fallingdotseq$ & స్వ్ & * 赎 & ฮ్రి & $\stackrel{\text { జ }}{0}$ & 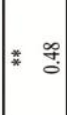 & $* \frac{\pi}{8}$ & * & $*$ ํํㅇ & * * : & 慗 & 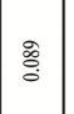 & డ్రి & छ్రి & 㑒 & * & 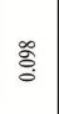 & స్తి & 莺 & $\stackrel{8}{\circ}$ \\
\hline 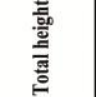 & $*$ 吉 & - & छे & $\frac{n}{3}$ & \# స & \begin{tabular}{|l|} 
\\
\end{tabular} & * aे & * & $\bar{\Xi}$ & * & $\approx$ : & శ్రి & : & $\stackrel{2}{\circ}$ & $\Xi$ & $\bar{\delta}$ & $\frac{5}{0}$ & * & $\stackrel{\infty}{\stackrel{\infty}{0}}$ & $\stackrel{2}{\tilde{z}}$ & : & לू. & ఫ్̊ㅇ \\
\hline 嘉 & - & $=\frac{1}{5}$ & $\bar{\partial}$ & : & 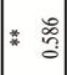 & 害 & * & * & 营 & ఫิ & * & 旁 & $\stackrel{\circ}{\circ}$ & * $\begin{array}{ll}* \\
\vdots\end{array}$ & ন্ন & * & * $\begin{array}{ll}0 \\
\vdots \\
0\end{array}$ & 瓷 & $\frac{n}{0}$ & gे & $\Xi$ & $=\frac{0}{0}$ & g̊ \\
\hline 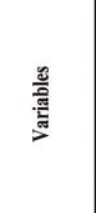 & 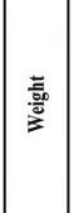 & 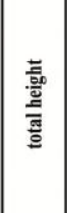 & 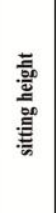 & 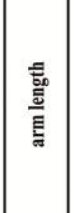 & 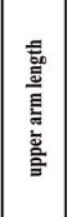 & 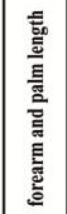 & 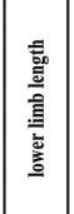 & 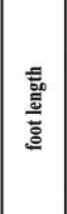 & 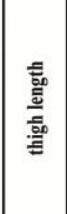 & 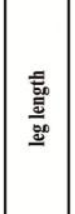 & 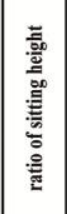 & 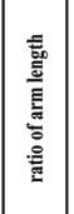 & 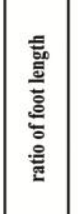 & 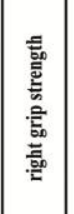 & 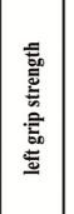 & 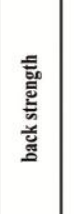 & 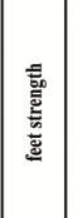 & 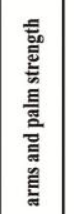 & 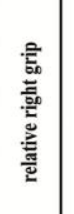 & 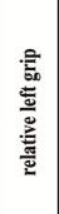 & 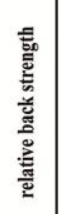 & 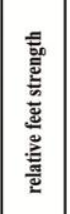 & 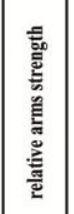 \\
\hline
\end{tabular}

Table (2) shows that simple correlation coefficients could be found in the form of matrix of correlation between the study variables. There are 121 correlation coefficients significant at $0.01,68$ significant at 0.5 and 340 insignificant. 
Table (3)

Factors matrix before rotation of the study variables

\begin{tabular}{|c|c|c|c|c|c|c|}
\hline \multirow{2}{*}{ Variables } & \multicolumn{6}{|c|}{ Factors } \\
\hline & 1 & 2 & 3 & 4 & 5 & 6 \\
\hline weight & 0.526 & 0.391 & 0.666 & 0.002 & 0.104 & 0.053 \\
\hline total height & 0.216 & 0.694 & 0.573 & 0.312 & 0.142 & 0.021 \\
\hline sitting height & 0.333 & 0.405 & 0.051 & 0.712 & 0.329 & 0.182 \\
\hline arm length & 0.041 & 0.712 & 0.460 & 0.322 & 0.144 & 0.094 \\
\hline upper arm length & 0.461 & 0.556 & 0.184 & 0.579 & 0.145 & 0.162 \\
\hline forearm and palm length & 0.263 & 0.670 & 0.468 & 0.156 & 0.403 & 0.235 \\
\hline lower limb length & 0.027 & 0.840 & 0.532 & 0.013 & 0.057 & 0.018 \\
\hline foot length & 0.029 & 0.814 & 0.303 & 0.154 & 0.359 & 0.299 \\
\hline thigh length & 0.817 & 0.357 & 0.010 & 0.149 & 0.193 & 0.312 \\
\hline leg length & 0.197 & 0.828 & 0.275 & 0.157 & 0.192 & 0.104 \\
\hline ratio of sitting height & 0.035 & 0.530 & 0.555 & 0.338 & 0.058 & 0.470 \\
\hline ratio of arm length & 0.153 & 0.431 & 0.769 & 0.046 & 0.068 & 0.320 \\
\hline ratio of foot length & 0.292 & 0.403 & 0.211 & 0.125 & 0.693 & 0.416 \\
\hline right grip strength & 0.876 & 0.195 & 0.176 & 0.159 & 0.252 & 0.130 \\
\hline left grip strength & 0.860 & 0.073 & 0.288 & 0.039 & 0.331 & 0.210 \\
\hline back strength & 0.909 & 0.104 & 0.028 & 0.070 & 0.130 & 0.296 \\
\hline feet strength & 0.751 & 0.047 & 0.347 & 0.497 & 0.113 & 0.119 \\
\hline arms and palm strength & 0.634 & 0.615 & 0.249 & 0.048 & 0.259 & 0.121 \\
\hline relative right grip & 0.761 & 0.444 & 0.113 & 0.200 & 0.351 & 0.148 \\
\hline relative left grip & 0.702 & 0.051 & 0.515 & 0.011 & 0.376 & 0.205 \\
\hline relative back strength & 0.813 & 0.032 & 0.339 & 0.069 & 0.141 & 0.364 \\
\hline relative feet strength & 0.702 & 0.154 & 0.187 & 0.608 & 0.154 & 0.147 \\
\hline relative arms strength & 0.417 & 0.454 & 0.606 & 0.039 & 0.258 & 0.165 \\
\hline latent root & 7,235 & 5,737 & 3,726 & 1,968 & 1,684 & 1,243 \\
\hline variance ratio $\%$ & 31,457 & 24,942 & 16,200 & 8,557 & 7,320 & 5,406 \\
\hline
\end{tabular}

Table (3) shows that 6 factors could be drawn according to the Kaiser test as variables were saturated on them. Combined variance ratio of 93.883 could be drawn for the population from the total variance among variables. Latent root was 7.235 for the first factor, and 1.243 for the sixth factor. 
Table (4)

Factors matrix after orthogonal rotation of the study variables

\begin{tabular}{|c|c|c|c|c|c|c|}
\hline \multirow{2}{*}{ Variables } & \multicolumn{6}{|c|}{ Factors } \\
\hline & 1 & 2 & 3 & 4 & 5 & 6 \\
\hline weight & 0.130 & 0.803 & 0.152 & 0.404 & 0.164 & 0.107 \\
\hline total height & 0.093 & 0.974 & 0.082 & 0.063 & 0.022 & 0.062 \\
\hline sitting height & 0.203 & 0.071 & 0.175 & 0.027 & 0.409 & 0.825 \\
\hline arm length & 0.063 & 0.283 & 0.800 & 0.192 & 0.211 & 0.261 \\
\hline upper arm length & 0.455 & 0.789 & 0.217 & 0.147 & 0.011 & 0.198 \\
\hline forearm and palm length & 0.332 & 0.065 & 0.877 & 0.125 & 0.078 & 0.270 \\
\hline lower limb length & 0.251 & 0.897 & 0.165 & 0.089 & 0.140 & 0.268 \\
\hline foot length & 0.096 & 0.752 & 0.143 & 0.045 & 0.631 & 0.035 \\
\hline thigh length & 0.676 & 0.014 & 0.061 & 0.331 & 0.590 & 0.178 \\
\hline leg length & 0.186 & 0.376 & 0.670 & 0.072 & 0.363 & 0.333 \\
\hline ratio of sitting height & 0.061 & 0.621 & 0.035 & 0.056 & 0.102 & 0.724 \\
\hline ratio of arm length & 0.072 & 0.223 & 0.853 & 0.287 & 0.044 & 0.209 \\
\hline ratio of foot length & 0.034 & 0.009 & 0.116 & 0.144 & 0.955 & 0.120 \\
\hline right grip strength & 0.817 & 0.236 & 0.248 & 0.340 & 0.155 & 0.128 \\
\hline left grip strength & 0.902 & 0.059 & 0.228 & 0.329 & 0.031 & 0.072 \\
\hline back strength & 0.460 & 0.224 & 0.164 & 0.761 & 0.037 & 0.280 \\
\hline feet strength & 0.262 & 0.190 & 0.194 & 0.875 & 0.174 & 0.176 \\
\hline arms and palm strength & 0.240 & 0.383 & 0.674 & 0.507 & 0.011 & $\square 100$ \\
\hline relative right grip & 0.903 & 0.124 & 0.236 & 0.177 & 0.120 & 0.203 \\
\hline relative left grip & 0.887 & 0.193 & 0.285 & 0.179 & 0.082 & 0.023 \\
\hline relative back strength & 0.455 & 0.086 & 0.290 & 0.698 & 0.011 & 0.384 \\
\hline relative feet strength & 0.227 & 0.031 & 0.141 & 0.915 & 0.150 & 0.188 \\
\hline relative arms strength & 0.179 & 0.004 & 0.807 & 0.346 & 0.071 & 0.188 \\
\hline latent root & 7.235 & 5.737 & 3.726 & 1.968 & 1.684 & 1.243 \\
\hline variance ratio $\%$ & 31.457 & 24.942 & 16.200 & 8.557 & 7.320 & 5.406 \\
\hline
\end{tabular}

Table (4) shows the results of factorial analysis after orthogonal rotation of the factor. 6 factors could be drawn just as they were in the analysis before rotation.
The table also shows that the factor number (1) was saturated by 9 variables. Their saturation ranged between 0.903 and 0.332 .

Table (5)

Saturated variables on the first factor

\begin{tabular}{|c|c|}
\hline Variable & Saturation \\
\hline relative right grip strength & 0.903 \\
\hline left grip strength & 0.902 \\
\hline relative left grip strength & 0.887 \\
\hline right grip strength & 0.817 \\
\hline thigh length & 0.676 \\
\hline back strength & 0.460 \\
\hline upper arm length & 0.455 \\
\hline relative back strength & 0.455 \\
\hline forearm and palm length & 0.332 \\
\hline
\end{tabular}

Table (5) shows that the first factor was saturated in 9 variables. The most saturated variable was the relative right grip strength with a saturation coefficient of 0.903 . The grip muscular strength factor prevailed over most of the saturated variables. Thus, we can call the first factor the grip strength factor. Factorial validity coefficient of this factor was 0.903 . 
It is evident from table (4) on factors matrix after rotation that the factor number (2) was saturated in 8 variables. Their saturation ranged between 0.974 and 0.376 .

Table (6)

Variables saturated in the second factor

\begin{tabular}{|c|c|}
\hline Variable & Saturation \\
\hline total height & 0.974 \\
\hline lower limb length & 0.897 \\
\hline weight & 0.803 \\
\hline upper arm length & 0.789 \\
\hline foot length & 0.752 \\
\hline ratio of sitting height & 0.621 \\
\hline arms and palm strength & 0.383 \\
\hline leg length & 0.376 \\
\hline
\end{tabular}

Table (6) shows that the second factor was saturated in 8 variables. The highest saturated variable was the total height with a saturation coefficient of 0.974 . Lengths variable factor prevailed over most of the saturated variables. Thus, we can call the second factor the lengths factor, represented by the total height of the stature. Factorial validity coefficient of this factor was 0.974 .

It is evident from table (4) of factors matrix after rotation that the factor number (3) was saturated in 6 variables. Their saturation ranged between 0.877 and 0.670 .

Table (7)

Variables Saturated in the third factor

\begin{tabular}{|c|c|}
\hline Variable & Saturation \\
\hline forearm and palm length & 0.877 \\
\hline ratio of arm length & 0.853 \\
\hline relative arms strength & 0.807 \\
\hline arm length & 0.800 \\
\hline arms and palm strength & 0.674 \\
\hline leg length & 0.670 \\
\hline
\end{tabular}

Table (7) shows that the third factor was saturated in 6 variables. The highest saturated variable was the forearm and palm length with a saturation coefficient of 0.877 . The limbs length variable prevailed over most of the saturated variables. Thus, we can call the third factor the upper limb length, represented by the forearm and palm length. Factorial validity coefficient of this factor was 0.670 .

It is evident from table (4) of factors matrix after rotation that the factor number (4) saturated 10 variables. Their saturation ranged between 0.915 and 0.329 .

Table (8)

Variables saturated in the fourth factor

\begin{tabular}{|c|c|}
\hline Variable & Saturation \\
\hline relative feet strength & 0.915 \\
\hline feet strength & 0.875 \\
\hline back strength & 0.761 \\
\hline relative back strength & 0.698 \\
\hline arms and palm strength & 0.507 \\
\hline total height & 0.404 \\
\hline relative arms strength & 0.346 \\
\hline right grip strength & 0.340 \\
\hline thigh length & 0.331 \\
\hline left grip strength & 0.329 \\
\hline
\end{tabular}

Table (8) shows that the fourth factor was saturated in 10 variables. The highest saturated was the variable of feet strength with a saturation coefficient of 0.915 . The maximum 
strength variables prevail over most of the saturated variables. Thus, we can call the fourth factor the strength factor, represented by the relative maximum strength of feet. The factorial validity coefficient was 0.915 .
It is evident from table (4) of factors matrix after rotation that the factor number (5) saturated 5 variables. Their saturation ranged between 0.955 and 0.363 .

Table (9)

Variables saturated in the fifth factor

\begin{tabular}{|c|c|}
\hline Variable & Saturation \\
\hline ratio of foot length & 0.955 \\
\hline foot length & 0.631 \\
\hline thigh length & 0.590 \\
\hline sitting height & 0.409 \\
\hline leg length & 0.363 \\
\hline
\end{tabular}

Table (9) shows that the fifth factor was saturated in 5 variables. The highest saturated variable was the ratio of foot length with a saturation coefficient of 0.955 . The lower limb length variable prevailed over most of the saturated variables. Thus, we can call the fifth factor the lower limb length, represented by the ratio of the foot length. The validity coefficient of this factor was 0.955 .

It is evident from table (4) of factors matrix after rotation that the factor number (6) saturated (4) variables. Their saturation ranged between 0.825 and 0.333 .

Table (10)

Variables saturated in the (6) sixth factor

\begin{tabular}{|c|c|}
\hline Variable & Saturation \\
\hline sitting height & 0.825 \\
\hline ratio of sitting height & 0.724 \\
\hline relative back strength & 0.384 \\
\hline leg length & 0.333 \\
\hline
\end{tabular}

Table (10) shows that the fifth factor was saturated in 4 variables. The highest saturated variable was sitting height variable. Its saturation coefficient was 0.825 . Sitting height variable prevailed over most saturated variables on the sixth factor. Thus, we can called factor number (6) factor of sitting height. The factorial validity coefficient was 0.825 .

Table (11)

The six final factors and their Factorial validity coefficients

\begin{tabular}{|c|c|c|}
\hline S & Factor & Factorial validity coefficient \\
\hline 1 & grip strength factor & 0.903 \\
\hline 2 & length factor & 0.974 \\
\hline 3 & upper limb length factor & 0.877 \\
\hline 4 & maximum strength factor & 0.915 \\
\hline 5 & lower limb length factor & 0.955 \\
\hline 6 & sitting height factor & 0.825 \\
\hline
\end{tabular}


Table (12)

Standard scores and the equivalent values for the extracted factors

\begin{tabular}{|c|c|c|c|c|c|c|}
\hline \multirow{2}{*}{$\begin{array}{c}\text { Standard } \\
\text { score }\end{array}$} & Total height & $\begin{array}{c}\text { Sitting } \\
\text { height }\end{array}$ & $\begin{array}{c}\text { Forearm } \\
\text { and palm } \\
\text { length }\end{array}$ & $\begin{array}{c}\text { Ratio of } \\
\text { foot length }\end{array}$ & $\begin{array}{c}\text { Relative } \\
\text { right grip }\end{array}$ & $\begin{array}{c}\text { Relative } \\
\text { feet } \\
\text { strength }\end{array}$ \\
\hline 10 & 167.5500 & 82.3000 & 44.1500 & 48.5170 & 0.4600 & 1.0030 \\
\hline 20 & 168.0000 & 85.2000 & 45.6000 & 49.9860 & 0.4680 & 1.1440 \\
\hline 25 & 168.0000 & 86.0000 & 46.0000 & 51.6500 & 0.5000 & 1.2400 \\
\hline 30 & 168.1500 & 86.0000 & 46.3000 & 51.6620 & 0.5030 & 1.2910 \\
\hline 40 & 169.5000 & 86.8000 & 48.0000 & 51.7140 & 0.5260 & 1.4380 \\
\hline 50 & 171.2500 & 88.0000 & 49.7500 & 51.8450 & 0.5550 & 1.4900 \\
\hline 60 & 172.7000 & 88.3000 & 50.0000 & 52.3840 & 0.5660 & 1.5000 \\
\hline 70 & 176.6500 & 88.8500 & 50.7000 & 52.6800 & 0.5770 & 1.5070 \\
\hline 75 & 178.0000 & 89.0000 & 51.0000 & 52.6800 & 0.5800 & 1.5100 \\
\hline 80 & 181.2000 & 89.0000 & 51.8000 & 52.7360 & 0.5800 & 1.5820 \\
\hline 90 & 182.0000 & 89.9000 & 53.8000 & 53.0830 & 0.6610 & 1.9060 \\
\hline
\end{tabular}

\section{Discussion:}

According to table (1), the arithmetic means of the total height of the body, the sitting height, the arm length, the upper arm length, the forearm and palm length, the lower limb length, the foot length, the thigh length, the leg length, the ratio of sitting height, the ratio of arm length, and the ratio of foot length in the sample subjects were, respectively $173.000,87.15$, 83.20, 33.85, 48.90, 86.15, 89.30, 35.90, 45.95, $51.12,47.70$, and 51.62 .

The same table shows that the arithmetic means of the strength variable, as represented in the right grip strength, the left grip strength, the back strength, the legs strength, the arms and palm strength, the relative right grip strength, the relative left grip strength, the relative back strength, the relative legs strength and the relative arms strength in the sample subjects were, respectively $39.40,37.20,93.50,104.00$, 96.50, 0.544, 0.516, 1.28, 1.429, and 1.334.

This is in line with results reached by Essam Abd el-Khalek (1987) about the relationship between the physical composition of the player in terms of height, weight and limb length and the possibility of attaining higher levels. Each sport activity requires specific physical characteristics that should be considered when selecting players for the various activities.

It also agrees with the findings of Mohammad Ali Mahmoud, Badr Mahmoud Shehata and Yasser Mahrous (2004), and Ali Fahmy AlBeik, and Sayed Abdel-Gawad (1980) on the fact that physical measurements are important factors that can not be ignored if the player is to reach higher levels of activity. (3) (10) (4).

Mohammed Sabri Omar (1981) also found that height, total height, upper arm length, forearm and the palm length, lower limb length and foot and leg lengths are the most important physical measurements for rowers. Omar also found that there is a direct relationship between the speed of the boat and the absolute and relative grip strength and the strength of leg muscles. He found a relationship between the rhythm of the strike and absolute and relative left and right grip strength and absolute and relative strength of leg muscles. (8).

The factorial analysis illustrated in tables (3) and (4) resulted in the following six saturation factors, with each factor saturated by a set of inter-related variables. The body measurements and strength variables saturated to a high or acceptable degree in a particular factor were very important to rowers seeking to reach higher levels. Less saturated measurements contributed less towards that goal. They were looked upon just as contributing measurements for rowers to reach higher levels.

\section{The first factor (grip strength):}

Table (5), illustrating, in a descending order, the major saturations of physical measurements and the strength variable of the grip strength, shows that 9 variables were saturated, excluding variables whose saturation was less than \pm 3 . The values of saturation in this factor ranged between 903 and 332 . 


\section{The second factor (height and length):}

Table (6), illustrating, in a descending order, the major saturations of physical measurements and the strength variable of height and length, shows that 8 variables were saturated, excluding variables whose saturation was less than \pm 3 . The values of saturation in this factor ranged between 974 and 376 .

\section{The third factor (the upper limb length):}

Table (7), illustrating, in a descending order, the major saturations of physical measurements and the strength variable of the upper limb length, shows that 6 variables were saturated, excluding variables whose saturation was less than \pm 3 . The values of saturation in this factor ranged between 877 and 670 .

\section{The fourth factor (maximum strength):}

Table (8) illustrating, in a descending order, the major saturations of physical measurements and the strength variable of maximum strength, shows that 10 variables were saturated, excluding variables whose saturation was less than \pm 3 . The values of saturation on this factor ranged between 915 and 329 .

\section{The fifth factor (the lower limb length):}

Table (9), illustrating, in a descending order, the major saturations of physical measurements and the strength variable of lower limb length, shows that 6 variables were saturated, excluding variables whose saturation was less than \pm 3 . The values of saturation on this factor ranged between 955 and 363 .

\section{The sixth factor (sitting height):}

Table (10), illustrating, in a descending order, the major saturations of physical measurements and the strength variable of sitting height, shows that 4 variables were saturated, excluding variables whose saturation was less than \pm 3 . The values of saturation on this factor ranged between 825 and 333 .

\section{Conclusions:}

Six factors were found to determine the physical characteristics of the high level rowers:

1. The grip strength factor, represented by the relative grip strength with a factorial validity coefficient of 0.903 .
2. The height factor, represented by the total height of the stature with a factorial validity coefficient of 0.974 .

3. The upper limb length factor, represented by the length of the forearm and palm with a factorial validity coefficient of 0.877 .

4. The maximum strength factor, represented by the relative maximum strength of the two legs with a factorial validity coefficient of 0.915 .

5. The lower limb length factor, represented by the ratio of the leg length with a factorial validity coefficient of 0.955 .

6. The sitting height factor with a factorial validity coefficient of 0.825 .

* Developing a standard scale for the standard grades of the measurements extracted.

\section{Recommendations:}

1. The author of this paper recommends using the selected battery of physical measurements for rowers to develop the activity through:

a. Improving the selection by using the battery and the standard grades extracted.

b. Upgrading training operations by improving the level of maximum strength, especially for the legs. Physical and kinanthropometric measurement must establish a relationship between the measured variables and actual rowing performance to substantiate a statement as this.

2. When selecting rowers, the following measurements should be considered:

(height - sitting height - forearm and palm length - ratio of the legs length - the grip strength - the maximum strength of the legs).

3. Training should increase the relative strength of the muscles of the body through muscle strength training, especially for the legs, raising the level of the relative strength of the body as a whole. 


\section{References:}

\section{Arabic References:}

1. Osama Kamel Rateb, Ali Mohammed Zaki: The Scientific Foundations of Swimming Training, Dar Al-Fikr Al-Arabi, 2ed., Cairo, 1992.

2. Esam Amin Helmy: A Comparative Study of Short and Long Distances Swimmers in Some Biological Characteristics, Thesis for the Master's Degree, Faculty of Physical Education for Boys, Helwan University, Alexandria, 1975.

3. Essam Abd El-Khalek Moustafa: Sports Training (Theories and Applications), Dar elMa'aref, Alexandria, 1994.

4. Ali Fahmy Al-Beik, Sayed Abdel-Gawad: Morphological Measurements as a Basis for Selecting Junior Short Distances Swimmers, the Third Scientific Conference, Faculty of Physical Education for Boys, Alexandria, 1980.

5. Kamal Abd El-Hamid Ismail: Tests and Measurements of Physical Education, unpublished notes, 2003.

6. Mohammed Sobhi Hassanein: Evaluation and Measurement in Physical Education, Part 1, third edition, Dar Al-Fikr Al-Arabi, 1995.

7. Mohammed Sabri Omar: the Relation between Physical Measurements and Some Mechanical Aspects of the Stroke, unpublished thesis for the Master's Degree, Faculty of Physical Education for Boys, Alexandria, 1978.

8. Williams, Siberian, and Ropson : the Effect of Changing Some Parts of the Free Style Boats on some Mechanical Aspects of Rowing, dissertation for the Ph. D Degree, Faculty of Physical Education for Boys, Helwan University, 1981.

9. Mohammed Sabri Omar, Sanaa Hassan Aljubely: the Relative Importance of Physical Measurements and their Relation to Front Crawl Swimming Speed for Short Distances Female Swimmers, 1988.

10. Mohammed Ali Mahmoud, Badr Mahmoud Shehata, Yasser Mahroos Moustafa: Evaluating
Some Physical, Physiological and Posture Measurements, and Somatotype for Egyptian Football Team Junior Players, Theories and Applications Journal, Faculty of Physical Education for Boys, Alexandria, no. 52, 2004.

\section{Foreign References:}

1. Fell, j. w. and P. T. Gaffney (2001): Physiological Profiles Of Australian Surf Boat Rowers. J. Science and Medicine in Sport, 4 (2):188 -195,

2. G. J. Slater, A. J. Rice, I Mujika, A.G. Hahn, K. Sharpe, D.G. Jenkins (2004): Physique Traits of Light Weight Rowers and their Relationship to Competitive Success , Br. J. Sports Med 2005, 39:736 -741.doi: 10.1136 /bism. 2004

3. Hamid Arazi Hassan Faraji and Seid Mehdi Mohammadi(2011) Anthropometric and Physiological Profiles of Elite Iranian Junior Rowers, Middle- East Journal of Scientific Research 9(2):162 -166.

4. Hunsicker, P.(1974) Human Performance Factors, in Larson, L. A. (Ed.), Fitness, Health and Work Capacity New York and London.: Macmillan Publishing,

5. Keer, Deborah A., Ross,W.D. and Norton, Kevin and Hume, Patricia and Kagawaa, Masaharu,:(2007) Olymbic Lightweight and Open-Class ROWERS Possess Distinctive physical and proportionately characteristics. Jounal of Sports Sciences 25 (1): pp. 43 -53.

6. Mikuliae : (2008).Anthropometric and Physiological Profiles of Rowers of Varying Ages and Ranks Kinesiol., 40(1):80-88 .

7. Niels H. Secher, Stefanos Volianitis "Rowing: Olympic Handbook of Sports Medicine" Wiley - Blackwell inc., 2007.

8. Rodriguez F.A: (1986) Physical Structure of International Lightweight Rowers, in Reilly, j.Watkins, J.Borms (eds). Kinantherropometry II. E \&F, N Spon, London pp.255-261.

9. Secher N . H.,O.Vaage: (1983) Rowing Performance, a Matheamatical Model Based on Analysis of Body Dimensions as Exemplified 
by Body Weight, Eur. J. Appl. Physiol.52:88 93.

10. Shephard R.J :( 1998) Science and Medicine of Rowing , a Review . J.Sports Sci . 16:603620 .
11. S.Kaloupsis, G.C. Bogdanis, E.Dimakopoulou, M.Maridaki. : (2008) Anthropometric Characteristics and Somatotype of Young Greek Rowers, Biology of Sport, vol. 25 n. 1 